\title{
Optimization of IrDA IrLAP Link Access Protocol
}

\author{
Vasileios Vitsas and Anthony C. Boucouvalas, Fellow, IEEE
}

\begin{abstract}
The widespread installation of millions of Infrared Data Association (IrDA) infrared (IR) ports in mobile devices for wireless communication applications necessitates for throughput performance optimization of the IR links at the IrDA link access protocol (IrLAP) link layer. For IrDA connectivity, link-layer throughput optimization is important for any line bit-error rate (BER). The paper provides a mathematical model with which we derive a simple equation linking IrLAP throughput with physical and link-layer parameters. Simple equations for optimum values of window size and frame length for maximum link-layer throughput as a function of BER are derived. A study of the importance of parameters such as link minimum turnaround time and $\boldsymbol{F}$-timer time out period is presented. Finally, a protocol improvement that utilizes special supervisory frames ( $S$ frames) to pass transmission control is proposed to deal with delays introduced by $\boldsymbol{F}$-timer expiration. Results indicate that employing the special $S$ frame highly improves throughput performance when optimum window and frame-size values are implemented.
\end{abstract}

Index Terms-Infrared Data Association (IrDA), infrared (IR) links, optical wireless, wireless communications.

\section{INTRODUCTION}

$\mathbf{R}$ ECENT GROWTH on laptop computers and on portable devices, such as personal digital assistants (PDAs) and digital cameras, leads to an increasing demand for information transfer from or between portable devices [1]. Digital representation of information is expanding to new devices such as video and photocameras. New devices have "computer-like" capabilities for storing and retrieving information such as mobile phones and portable information gathering appliances. Computer manufacturers have adopted the Infrared Data Association (IrDA) standard [2] and almost every portable computer and all Windows CE devices on the market today contain an infrared (IR) port according to standards developed by IrDA. Laptop computers, PDAs, digital cameras, mobile phones, and printers are examples of devises with IrDA links. More than 40000000 devices are shipped each year with IrDA ports [3] capable of using the unregulated IR spectrum for their cable-less communication needs.

IrDA standard addresses low-cost, indoor, short range, half duplex, point-to-point links [4]. The IrDA physical layer specification (Ir-PHY) [5] supports optical links from 0 to at least

\footnotetext{
Manuscript received July 23, 2001; revised April 26, 2002 and May 14, 2002; accepted June 3, 2002. The editor coordinating the review of this paper and approving it for publication is W. W. Lu.

V. Vitsas is with the Multimedia Communications Group, School of Design, Engineering and Computing, Bournemouth University, Dorset BH12 5BB, U.K., on leave from the Department of Information Technology, Technological Educational Institution, Thessaloniki, Greece (e-mail: vvitsas@bournemouth.ac.uk).

A. C. Boucouvalas is with the Multimedia Communications Group, School of Design, Engineering and Computing, Bournemouth University, Dorset BH12 5BB, U.K. (e-mail: tboucouv@bournemouth.ac.uk).

Digital Object Identifier 10.1109/TWC.2003.816776
}

$1 \mathrm{~m}$, an angle of $\pm 15^{\circ}$ at a bit-error rate (BER) of less that $10^{-8}$. Ir-PHY ver 1.0 serial IR (SIR) specification [6] supported data rates up to $115.2 \mathrm{~kb} / \mathrm{s}$ using standard serial hardware, Ir-PHY ver 1.1 fast IR (FIR) [7] extended data rate to $4 \mathrm{Mb} / \mathrm{s}$, and finally Ir-PHY ver 1.3 very fast IR (VFIR) [8] specification added the $16-\mathrm{Mb} / \mathrm{s}$ link rate. The IrDA hardware is controlled by a link-layer protocol, the IrDA link access protocol (IrLAP) [9], [10]. IrLAP is based on the widely used high-level data link control (HDLC) protocol operating in normal response mode (NRM). The performance of IrDA optical wireless links may be measured by the throughput which can be drawn at the IrLAP layer.

In this paper, we concentrate on the performance evaluation of the IrLAP protocol and on deriving optimum values for link-layer parameters for maximizing throughput. In the literature, a mathematical model for the IrLAP throughput using the concept of a frame's "virtual transmission time" is presented in [11] and [12] based on the HDLC analysis model presented in [13]. However, this model does not lead to a simple formula for the IrLAP throughput. In this work, a new mathematical model using the average window transmission time (WTT) is developed. By taking advantage of IrLAP half duplex operation, this model leads to a simple closed form formula for IrLAP throughput. The formula relates throughput with physical layer parameters, such as link BER, link data rate, and minimum turnaround time, and with link-layer parameters such as frame size, window size, and frame overhead. As this equation gives us an intuitive understanding of the performance of IrDA links, it would be very valuable for designers and implementers of such links. By setting the first derivative equal to zero, we derive the optimum values for window size and frame length that maximize throughput. Formulas for all IrLAP time-consuming tasks are also presented, allowing evaluation of link parameter values to throughput performance. IrLAP performance is examined for various link parameters, such as BER, data rate, and window size and compared with optimum performance achieved by using optimum window size and frame length values. Optimum window and frame-size values can be easily implemented and result in significant throughput increase, especially for links experiencing high BERs. However, implementing optimum frame-size values on retransmissions requires buffer reorganization at a low level.

The paper is outlined as follows. Section II presents the IrLAP layer procedures. The parameter definitions and the information transfer model employed for IrLAP throughput evaluation is discussed in Section III, and Section IV presents a mathematical model that uses the average WTT to evaluate IrLAP throughput and the other IrLAP time-consuming tasks. The IrLAP throughput performance is analyzed in Section V in relation to physical and link-layer parameters. Optimum 


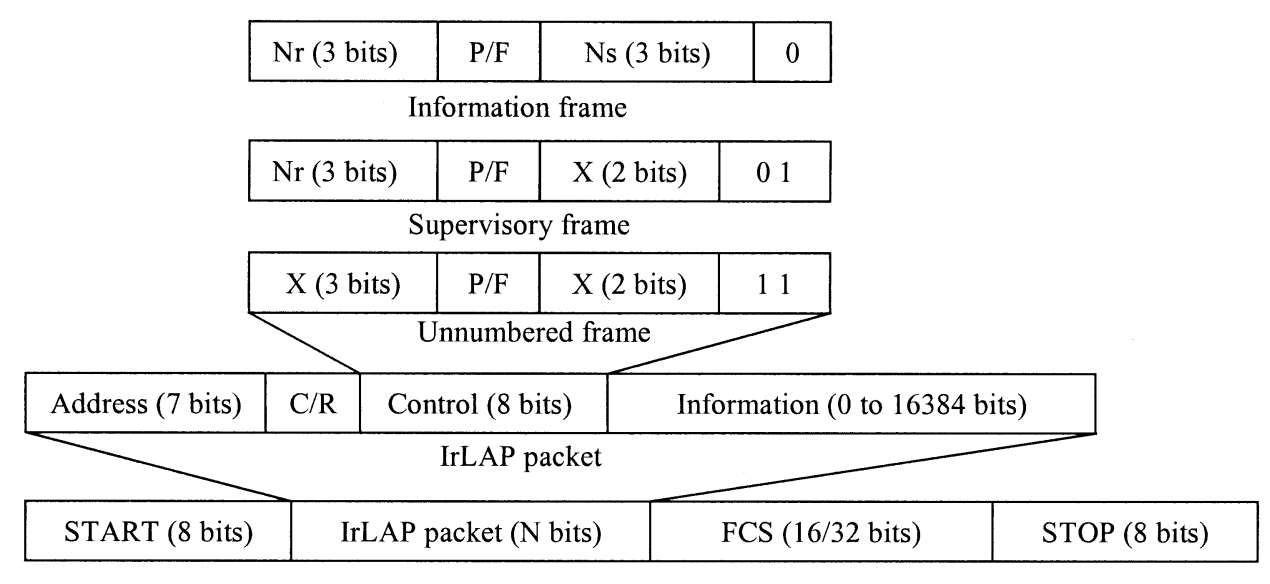

Physical layer frame

Fig. 1. IrDA SIR and FIR frame structure.

window and frame-size values for maximum throughput are derived in Section VI. This section also validates the mathematical analysis for maximum throughput performance by comparing results obtained by equations for optimum window and frame-size values with results obtained by employing numerical methods for maximum IrLAP throughput. Throughput performance achieved by employing optimum values for window and/or frame size is presented in Section VII, and concluding remarks are given in Section VIII.

\section{THE IrLAP LAYER}

IrLAP is the IrDA data link layer. It is designed based on the pre-existing HDLC and synchronous data link control protocols [9]. IrLAP stations operate in two modes: in the normal disconnect mode during the contention period and in the NRM during the connection period. In the contention period, a station advertises its existence to the neighboring stations along with the link parameters it supports and wishes to employ during the connection period. One of the participating stations becomes the primary station. Any station may claim to become the primary station but, at the end of the contention period, only one station is granted the primary role and all other stations are assigned the secondary role. All data traffic during the connection period are sent to or from the primary station. A secondary station wishing to communicate to another secondary station does so through the primary station. The parameters negotiated and agreed on during the contention period are given below.

1) Data rate $(C)$. This parameter specifies the station's transmission rate.

2) Maximum turnaround time $\left(T_{\max }\right)$. This parameter specifies the maximum time interval a station can hold transmission control. For data rates less than $115.2 \mathrm{~kb} / \mathrm{s}$, the maximum turnaround time must be $500 \mathrm{~ms}$. A smaller value may be agreed to between the two stations for $115.2 \mathrm{~kb} / \mathrm{s}$ or higher data rates.

3) Data size ( $l$ ). This is the maximum length allowed for the data field in any received information frame ( $I$ frame). This parameter has an upper limit of 2048 bytes $(16 \mathrm{~kb})$.

4) Window size $\left(W_{\max }\right)$. This is the maximum number of unacknowledged $I$ frames a station can receive before it has to acknowledge the number of $I$ frames received correctly. An acknowledgement may be requested by the transmitting station before the window size is reached. This parameter has an upper limit of 7 for data rates up to $4 \mathrm{Mb} / \mathrm{s}$ and 127 for 4 and $16 \mathrm{Mb} / \mathrm{s}$ [9], [10].

5) Minimum turnaround time $\left(t_{\mathrm{ta}}\right)$. This is the time required by the station's receive circuit to recover after the end of a transmission initiated from the same station (turnaround latency). Each station must wait a minimum turnaround time delay when moving from receive mode to transmit mode to ensure that the receive circuit of the station that was transmitting is given enough time to recover. This is the time required to change link direction.

Both stations must use the same data rate. However, parameters (2), (3), (4), and (5) are negotiated and agreed independently for each station. The IrDA frame structure is shown in Fig. 1. frame check sequence (FCS) contains a 16-b cyclic redundancy check (CRC) for data rates up to $4 \mathrm{Mb} / \mathrm{s}$ and a 32-b $\mathrm{CRC}$ for $4-\mathrm{Mb} / \mathrm{s}$ and higher rates. IrLAP employs the following frame types.

1) Unnumbered frames (U frames) are used for link management. $U$ frames' functions include discovering and initializing secondary stations, reporting procedural errors not recoverable by retransmissions, etc.

2) I frames carry information data across the link during the connection period. I-frame control field contains send and receive frame counts to ensure ordered frame reception.

3) Supervisory frames ( $S$ frames) assist in information data transfer although $S$ frames never carry information data themselves. They are used to acknowledge correctly received frames, request an acknowledgement from the communicating station, convey station conditions, etc.

The control field contains an identifier, which determines the frame type. Depending on frame type, the control field may contain a send sequence number $N_{s}$ used to number the transmitted frames. It may also contain a receive sequence number $N_{r}$ used to indicate the expected sequence number of the next $I$ frame. SIR and FIR specifications employ an 8-b-long control field (Fig. 1). $N_{s}$ and $N_{r}$ occupy 3 b each in the control field, thus, $N_{s}$ 
and $N_{r}$ cycle through values from zero to seven and maximum window size is seven. VFIR specification extended the control field to $16 \mathrm{~b}$ for the $4-$ and $16-\mathrm{Mb} / \mathrm{s}$ data rate IrDA links. In this case, $N_{s}$ and $N_{r}$ occupy $7 \mathrm{~b}$ each, cycling through values 0 to 127 and a maximum window size of 127 is allowed.

Within the control field, the $P / F$ bit implements token passing between stations. When it is set by the primary station, it is the poll $(P)$ bit. When it is set by the secondary station, it is the final $(F)$ bit. Primary uses the $P$ bit to reverse link direction and solicit a response from the secondary. The secondary responds by transmitting one or more frames and by setting the $F$ bit of the last frame it transmits, thus, reversing link direction and returning transmission control to the primary. IrLAP primary and secondary stations also employ the $P$ timer. $P$ timer is assigned with the maximum turnaround time $\left(T_{\max }\right)$ agreed between stations during the contention period and represents the maximum time a station can hold transmission control. Each station starts the $P$ timer upon reception of a frame with the $P / F$ bit set and stops the $P$ timer when it transmits a frame with the $P / F$ bit set. If the $P$ timer expires, meaning that the station holds transmission control longer than allowed, the station immediately sends a receive ready (RR) $S$ frame with the $P / F$ bit set to pass transmission control. The primary station also employs an $F$ timer to limit the time a secondary station can hold transmission control. The primary starts the $F$ timer upon transmission of a frame with the $P$ bit set and stops the $F$ timer upon reception of a frame with the $F$ bit set. $F$-timer expiration means that the secondary failed to return transmission control within the agreed time period. Since the secondary's $P$-timer operation guarantees that this never happens, $F$-timer expiration can only be explained by the loss of either the frame containing the $P$ bit or the frame containing the $F$ bit. The primary resolves this situation by transmitting an RR frame with the $P$ bit set when the $F$ timer expires.

\section{IrLAP FunCtiONAL MODEL DESCRIPTION}

In this work, the transmission of a large amount of information data from the primary to the secondary station is considered. The saturation case is assumed, where the primary station always has information data ready for transmission.

The parameters used in the current model are shown in Table I. In the contention period, the primary station determines the window size $N$ it will employ. $N$ represents the maximum number of $I$ frames the primary can transmit before soliciting an acknowledgement. Maximum window-size parameter $W_{\max }$ is negotiated and agreed between the two stations during the contention period. However, the maximum time a station can hold transmission control $T_{\max }$ must always be obeyed and, according to IrLAP specification [9], $T_{\max }$ combined with frame length and link data rate may limit the window size applied. In other words, if the time needed for transmitting $W_{\text {max }}$ frames carrying "frame length" information bytes at the link data rate exceeds $T_{\max }$, then a smaller window size must be employed. Thus, $N$ is giver by

$$
N=\min \left\{W_{\text {max }}, \text { floor }\left(\frac{T_{\max }}{t_{I}}\right)\right\}
$$

TABLE I

ANALYSIS PARAMETERS

\begin{tabular}{l|l|l}
\hline Parameter & Description & Unit \\
\hline$C$ & Link data bit rate & bits /sec \\
\hline$p_{b}$ & Link bit error rate & - \\
\hline$p$ & Frame error probability & - \\
\hline$l$ & I-frame message data length & bits \\
\hline$l$ & S-frame length / I-frame overhead & bits \\
\hline$t_{I}$ & Transmission time of an I-frame & sec \\
\hline$t_{I m a x}$ & $\begin{array}{l}\text { Transmission time of an I-frame } \\
\text { with 16Kbits user data }\end{array}$ & sec \\
\hline$t_{S}$ & Transmission time of an S-frame & sec \\
\hline$t_{t a}$ & Minimum turn-around time & sec \\
\hline$t_{a c k}$ & Acknowledgement time & sec \\
\hline$T_{\max }$ & Maximum turn-around time & sec \\
\hline$t_{\text {Fout }}$ & F-timer time-out period & sec \\
\hline$W_{\max }$ & Maximum window size & frames \\
\hline$N$ & Window size & frames \\
\hline$D_{f}$ & Frame throughput & frames/sec \\
\hline$D_{b}$ & Data throughput & bits/sec \\
\hline & &
\end{tabular}

where min is "the lesser of" and floor is "the largest integer not exceeding." In this work, $T_{\max }$ is always fixed to $500 \mathrm{~ms}$.

The information transfer procedure used in the current model is presented in Fig. 2. Each node holds three variables, $V_{s}$ for counting $I$ frames transmitted, $V_{r}$ for counting $I$ frames received, and $w$ indicating the number of the remaining $I$ frames the station can transmit before reversing link direction. The primary also employs an $F$ timer for limiting the secondary's transmission period. When the primary station sends an $I$ frame, the $N_{s}$ subfield of the frame's control field is assigned the current $V_{s}$ value and $V_{s}$ is increased by one (modulo 8 or 128 depending of the control field size employed). The primary also makes a buffer copy of the frame for possible retransmissions. Since the primary always has information ready for transmission, it immediately checks the $w$ value. If $w$ is not equal to one, the primary reduces $w$ by one, transmits the $I$ frame with the $P$ bit not set and the actions previously described are repeated. When $w$ reaches one, indicating that the next $I$ frame should be the last frame in the window transmission, the primary sets the $P$ bit to poll the secondary and transmits the $I$ frame. The primary also assigns $N$ to $w$ for the next $N$ window frame transmission and starts the $F$ timer.

When the secondary station receives an $I$ frame, it compares the received frame sequence $N_{s}$ value with the expected $V_{r}$ value. If $N_{s}$ equals $V_{r}$ (the received frame is in sequence), $V_{r}$ is increased by 1 (modulo 8 or 128) and information data is extracted and passed to the upper layer. If the received frame is not in sequence (one of the previous $I$ frames in current window transmission was lost due to a CRC error), the frame is discarded and $V_{r}$ remains unchanged. The secondary station also checks the $P$ bit. If the $P$ bit is set and, as the current model assumes that the secondary station never has information for transmission, it awaits a minimum turnaround time $t_{\mathrm{ta}}$ to allow for the hardware recovery latency and transmits an $S$ frame with the $F$ bit set. The $S$ frame's $N_{r}$ field contains $V_{r}$, a value informing the primary of the number of $I$ frames received correctly and in sequence in the previous window transmission. When the primary receives the $S$ frame, it resumes 
P R IM A R Y

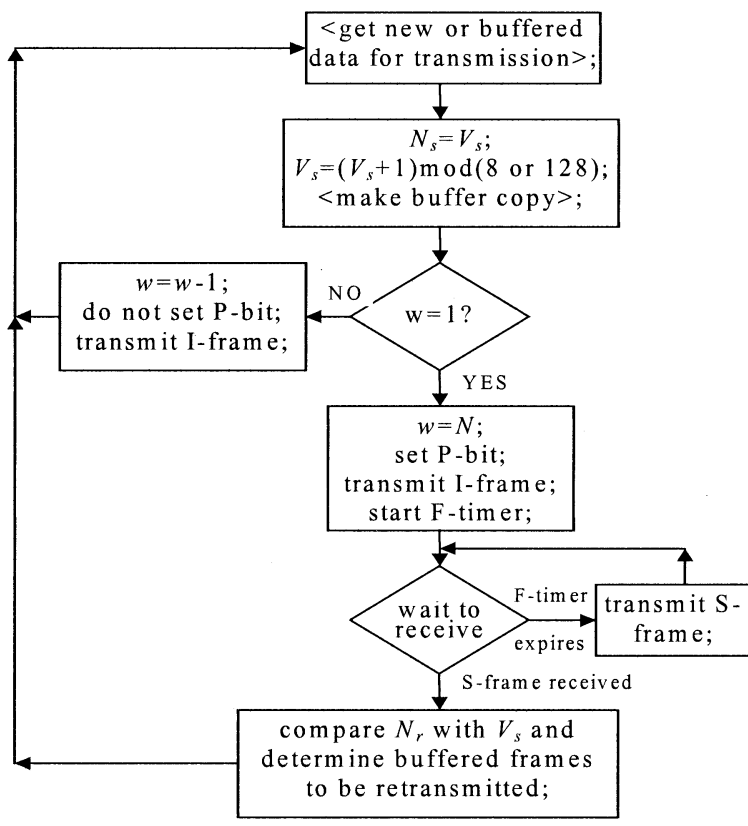

Fig. 2. Information transfer procedure.

$I$-frame transmission as transmission control was returned to the primary by means of the $F$ bit. The primary first compares the received $S$ frame's $N_{r}$ with its current $V_{s}$ value. If $N_{r}$ equals $V_{s}$ (all frames in the previous window transmission were received correctly by the secondary), the primary transmits $I$ frames containing new information data to the secondary. If $N_{r}$ is not equal to $V_{s}$, one or more $I$ frames in the previous window transmission are lost. The primary retransmits buffered $I$ frames starting from the indicated $N_{r}$ position before new data can be transmitted.

If the last $I$ frame that contains the $P$ bit is lost, the secondary station fails to respond as it does not realize that it has transmission control. The situation is resolved by the primary's F-timer expiration. The primary realizes that the secondary failed to respond within the agreed time period and transmits an $S$ frame forcing the secondary to respond. In the current model, $S$ frames are considered small enough to be always received error free.

The saturation case model considered in this work can be summarized as follows. The transmitting station always has information ready for transmission. As a result, it transmits a window of $N$ consecutive $I$ frames and reverses link direction by setting the $P$ bit in the last $I$ frame. The receiver awaits a minimum turnaround time and responds with an RR $S$ frame indicating the expected sequence number of the next frame. RR frames always have the $F$ bit set. The transmitter determines the number of frames correctly received before any error(s) occurred and repeats the erred frame and the frames following it, in the next window, followed by new frames to form a complete $N$ frame transmission. If the last frame in a window transmission is lost, the receiver fails to respond as the $P$ bit is lost. When $F$ timer expires, the primary station sends an RR $S$ frame with the $P$ bit set forcing the secondary station to acknowledge correctly received frames.
SECOND A R Y

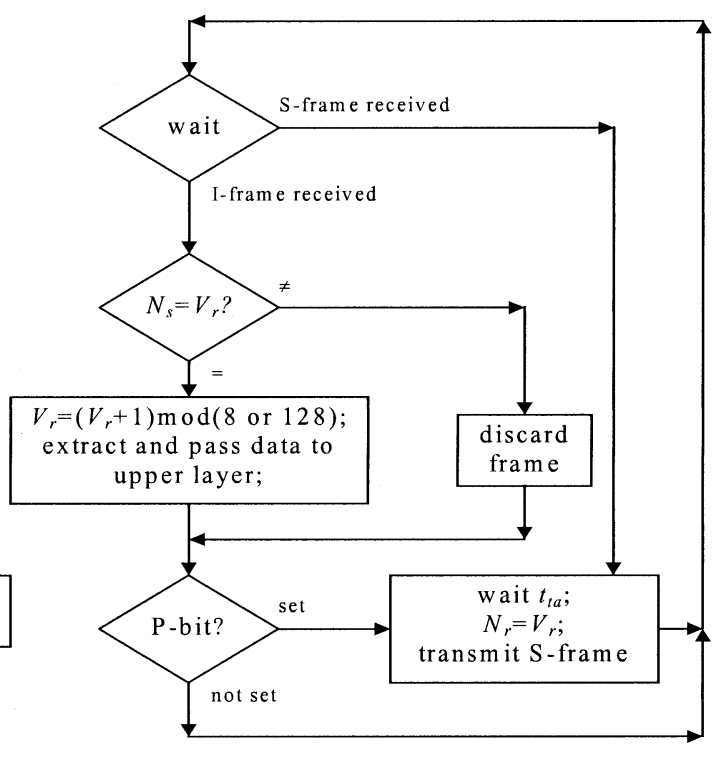

The values for $t_{S}, t_{I}, t_{\mathrm{ack}}, p$, and $D_{b}$ are given by (Fig. 3)

$$
\begin{aligned}
t_{S} & =\frac{l^{\prime}}{C} \\
t_{I} & =\frac{l+l^{\prime}}{C} \\
t_{\mathrm{ack}} & =2 t_{\mathrm{ta}}+t_{S} \\
p & =1-\left(1-p_{b}\right)^{l+l^{\prime}} \\
D_{b} & =l D_{f} .
\end{aligned}
$$

This model uses the term WTT to denote the average time needed for a complete window frame transmission and for acknowledgements and delays concerning this transmission. WTT accounts for the average time taken from the start of the first frame in a window transmission to the start of the first frame in the next window transmission. WTT incorporates time needed for $I$-frame transmissions, acknowledgements, reversing link direction, and time wasted in possible timer time out delays.

As shown in Fig. 3, the key issue that determines WTT is the reception of the last $I$ frame in the window, the frame that contains the $P$ bit. If this $I$ frame is correctly received, regardless of the existence of previous errors [Fig. 3(a) and (b)], WTT $t_{w}$ is given by

$$
t_{w}=N t_{I}+t_{\mathrm{ack}}
$$

If the $I$ frame containing the $P$ bit is lost, an additional delay for $F$-timer expiration and $S$-frame transmission $t_{s}$ is introduced. WTT is independent of possible additional $I$-frame errors. This situation is shown in Fig. 3(c) and WTT is given by

$$
t_{w}=N t_{I}+t_{\text {Fout }}+t_{s}+t_{\text {ack }}
$$




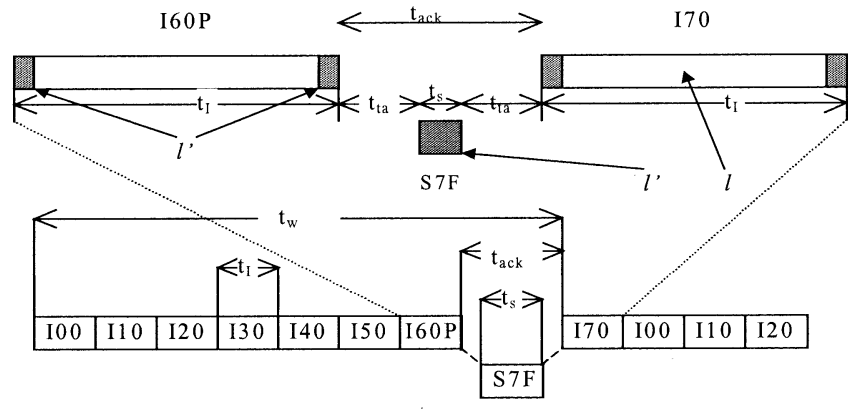

(a)

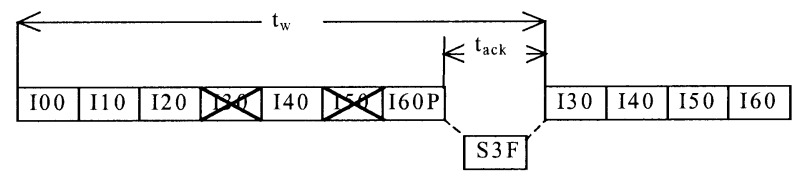

(b)

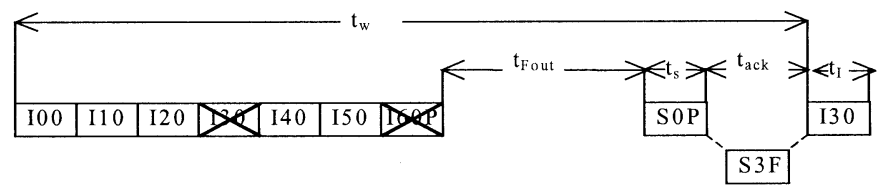

(c)

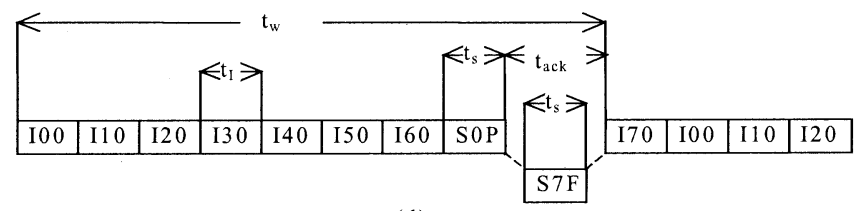

(d)

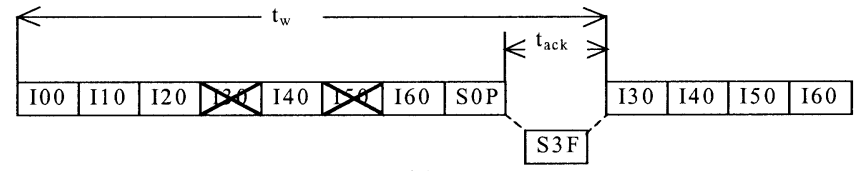

(e)

Fig. 3. Determination of WTT $t_{w} \cdot I_{x y}: I$ frame with $N_{s}=x$ and $N_{r}=y$. $I_{x y} P: I$ frame with $N_{s}=x, N_{r}=y$, and $P$ bit set. $S_{x} P: S$ frame with $N_{r}=x$ and $P$ bit set. $S_{x} F: S$ frame with $N_{r}=x$ and $F$ bit set. (a) Window error-free transmission. (b) Retransmitted frames due to error frame with $N_{s}=$ 3 and $N_{s}=5$. (c) Retransmitted frames and $F$-timer delay due frame error at $N_{s}=3$ and $N_{s}=6$. (d) Window error-free transmission ( $P$ bit in $S$ frame). (e) Retransmitted frames due to error frame with $N_{s}=3$ and $N_{s}=5$ ( $P$ bit in $S$ frame).

As an $I$ frame is incorrectly received with probability $p$, the average WTT is given by

$$
t_{w}=N t_{I}+p\left(t_{\text {Fout }}+t_{s}\right)+t_{\text {ack }} .
$$

Considering that all $I$ frames that follow an $I$ frame incorrectly received in an $N$ window frame transmission are considered as out of sequence and are discarded by the receiver, the probability $p_{c}(w)$ that exactly $w I$ frames at the beginning of a window transmission are correctly received followed by an error $I$ frame in position $(w+1)$ is

$$
p_{c}(w)=(1-p)^{w} p, \quad w=0,1,2, \ldots, N-1 .
$$

The probability that all $I$ frames in a window transmission are correctly received is

$$
p_{c}(N)=(1-p)^{N} .
$$

The expected number of correctly received frames, $p_{\text {all }}$, at the beginning of an $N I$ frame window transmission is

$$
p_{\text {all }}=\sum_{w=0}^{N} w p_{c}(w) .
$$

Frame throughput $D_{f}$ can now be found by dividing the expected number of $I$ frames $p_{\text {all }}$ correctly received in a window transmission by the average WTT required for the window transmission

$$
D_{f}=\frac{\sum_{w=0}^{N} w p_{c}(w)}{N t_{I}+p\left(t_{\text {Fout }}+t_{s}\right)+t_{\text {ack }}} .
$$

After some algebra, (13) reduces to

$$
D_{f}=\frac{1-p}{p} \frac{\left(1-(1-p)^{N}\right)}{N t_{I}+p\left(t_{\mathrm{Fout}}+t_{s}\right)+t_{\mathrm{ack}}}
$$

and by combining (6) with (14), link throughput is given by

$$
D_{b}=l \frac{1-p}{p} \frac{\left(1-(1-p)^{N}\right)}{N t_{I}+p\left(t_{\text {Fout }}+t_{s}\right)+t_{\text {ack }}} .
$$

An intuitive explanation of (14) is as follows. Term $(1-p) / p$ represents the expected number of frames correctly received before a frame error occurs. It counts for the frames from the first frame in a window transmission that follows a window containing an error to the next frame in error. Term $\left(1-(1-p)^{N}\right)$ is the probability that there is at least an error in a window transmission and term $N t_{I}+p\left(t_{\text {Fout }}+t_{s}\right)+t_{\text {ack }}$ stands for average WTT.

An extensive discussion on IrLAP mathematical models and a validation of the proposed WTT mathematical model in this paper can be found at [15]. This analysis allows for the evaluation of all component tasks affecting IrLAP throughput. Such an evaluation reveals the main factors resulting in throughput degradation for IrLAP operation in nonideal conditions. Equation (15) can be rewritten as

$$
D_{b}=l \frac{(1-p)}{p} \frac{\left(1-(1-p)^{N}\right)}{N \frac{l+l^{\prime}}{C}+p\left(t_{\text {Fout }}+t_{s}\right)+t_{\text {ack }}} .
$$

Time portion attributed to acknowledgements $T_{\text {tack }}$ is given by

$$
T_{\mathrm{tack}}=\frac{t_{\mathrm{ack}}}{N \frac{l+l^{\prime}}{C}+p\left(t_{\mathrm{Fout}}+t_{s}\right)+t_{\mathrm{ack}}} .
$$

Time portion used on $P$ bit loss and $F$-timer expiration $T_{\text {Fout }}$ is given by

$$
T_{\mathrm{Fout}}=\frac{p\left(t_{\mathrm{Fout}}+t_{s}\right)}{N \frac{l+l^{\prime}}{C}+p\left(t_{\mathrm{Fout}}+t_{s}\right)+t_{\mathrm{ack}}} .
$$

Time portion taken on transmitting frame overheads $l^{\prime}$ is given by

$$
T_{l^{\prime}}=\frac{\frac{N l^{\prime}}{C}}{N \frac{l+l^{\prime}}{C}+p\left(t_{\text {Fout }}+t_{s}\right)+t_{\text {ack }}} .
$$


TABLE II

$N t_{I}$ AND $t_{\text {ack }}$ FOR SIR AND FIR DATA RATES

\begin{tabular}{l|l|r|r|r|r|r|r}
\hline specification & data rate & year & $\begin{array}{c}\text { window } \\
\text { size } \\
\text { (frames) }\end{array}$ & $\begin{array}{c}\text { effective } N \\
\text { (frames) }\end{array}$ & $N t_{/}(\mathrm{ms})$ & $\begin{array}{r}t_{\text {ta }}(m s) \\
\text { (max) }\end{array}$ & $t_{\text {ack }}(\mathrm{ms})$ \\
\hline SIR & $115.2 \mathrm{Kbit} / \mathrm{s}$ & 1994 & 7 & 3 & 427.9 & 10 & 20.00 \\
\hline FIR & $576 \mathrm{Kbit} / \mathrm{s}$ & 1995 & 7 & 7 & 199.7 & 10 & 20.00 \\
\hline FIR & $1.152 \mathrm{Mbit} / \mathrm{s}$ & 1995 & 7 & 7 & 99.8 & 10 & 20.00 \\
\hline FIR & $4 \mathrm{Mbit} / \mathrm{s}$ & 1995 & 7 & 7 & 28.8 & 10 & 20.00 \\
\hline VFIR & $4 \mathrm{Mbit} / \mathrm{s}$ & 1999 & 127 & 121 & 497.8 & 10 & 20.00 \\
\hline VFIR & $16 \mathrm{Mbit} / \mathrm{s}$ & 1999 & 127 & 127 & 130.6 & 0.1 & 0.20 \\
\hline
\end{tabular}

As the expected number of error frames in a window transmission is $p N$, time portion spent on retransmission of error frames $T_{\text {error }}$ is

$$
T_{\text {error }}=\frac{\frac{p N l}{C}}{N \frac{l+l^{\prime}}{C}+p\left(t_{\text {Fout }}+t_{s}\right)+t_{\text {ack }}} .
$$

The expected number of correctly transmitted frames following an error frame in a window transmission can be found if from the total number of frames in a window $N$, we subtract the error frames $p N$ and the correct in sequence frames $(1-p)\left(1-(1-p)^{N}\right) / p$. Thus, the time portion spent on retransmitting correctly received out-of-sequence frames is given by

$$
T_{\text {corr }}=\frac{\left(N(1-p)-\frac{(1-p)}{p}\left(1-(1-p)^{N}\right)\right) \frac{l}{C}}{N \frac{l+l^{\prime}}{C}+p\left(t_{\text {Fout }}+t_{s}\right)+t_{\text {ack }}} .
$$

\section{ThoughPut ANalysis}

Equation (14) allows an intuitive understanding of IrLAP performance. Three factors contribute to average WTT given in (9). Factor $N t_{I}$ represents the user data transmission, factor $p\left(t_{\text {Fout }}+t_{s}\right)$ represents the lost $P / F$ bit overhead, and $t_{\text {ack }}$ the delays introduced by reversing link direction. It is clear that for very low BERs, factor $p\left(t_{\text {Fout }}+t_{s}\right)$ introduces negligible overhead as the $P / F$ bit is seldom lost. Table II shows $N t_{I}$ and $t_{\text {ack }}$ factors for IrPHY data rate evolution over the years. It presents the data rate(s) introduced by every new specification, the year the new specification was introduced, the specification's maximum window size ( $\left.W_{\max }\right)$, the maximum window size that can be enforced for 16-kb $I$ frames within $T_{\max }$ (effective $N$ ), specification's maximum $t_{\mathrm{ta}}$ value, and the two factors contributing to WTT. Table II reveals that the FIR specification introduced much higher data rates (up to $4 \mathrm{Mb} / \mathrm{s}$ ) without the expected change in the maximum $t_{\mathrm{ta}}$ value allowed for FIR IrDA ports. As a result, the time utilized for user data transmission dropped from 427.9 to $28.8 \mathrm{~ms}$ while the time spent on reversing the link direction twice was constant at $20 \mathrm{~ms}$ since $t_{\mathrm{ta}}$ was not changed. As a result, $4-\mathrm{Mb} / \mathrm{s}$ IrDA links employing minimum turnaround time $t_{\mathrm{ta}}=10 \mathrm{~ms}$ utilize $20 \mathrm{~ms}$ for acknowledgement for every $28.8 \mathrm{~ms}$ of user data transmission! Fig. 4 plots throughput efficiency versus BER for SIR and FIR link rates with $t_{\mathrm{ta}}=10 \mathrm{~ms}$, $W_{\text {max }}=7, l=16 \mathrm{~kb}$, and $t_{\text {Fout }}=t_{\text {Imax }}+2 t_{\text {ta }}$. Throughput efficiency decreases with data rate increase since link turnaround frequency is increased. As a result, a maximum throughput efficiency of 0.59 can be achieved for $4-\mathrm{Mb} / \mathrm{s}$ links.

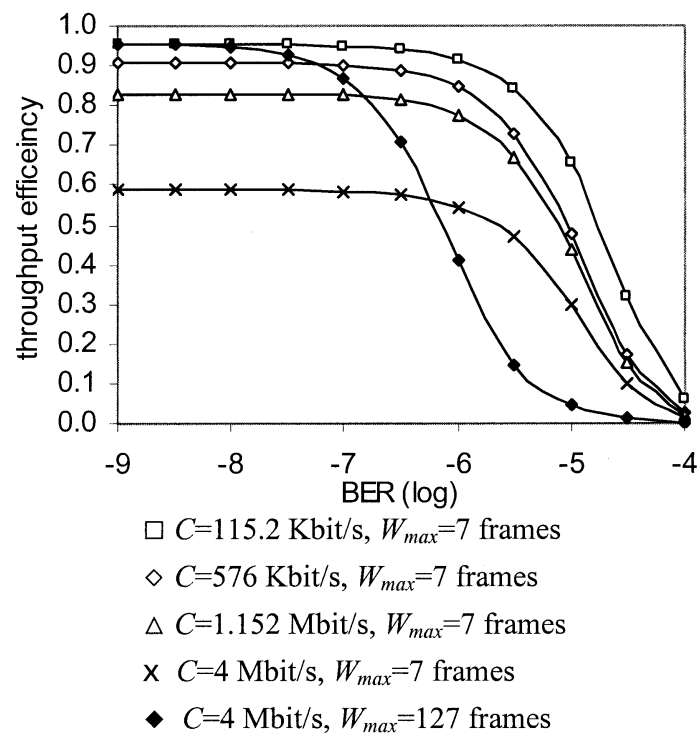

Fig. 4. Throughput efficiency versus BER for $t_{\mathrm{ta}}=10 \mathrm{~ms}, l=16 \mathrm{~kb}$, and $t_{\text {Fout }}=t_{\text {Imax }}+2 t_{\text {ta }}$.

VFIR specification, along with introducing the higher $16-\mathrm{Mb} / \mathrm{s}$ rate, addressed the problem by reducing maximum $t_{\mathrm{ta}}$ to $0.1 \mathrm{~ms}$ and by optionally increasing window size to 127 frames for $16-\mathrm{Mb} / \mathrm{s}$ links. VFIR also introduced an optional window size increase to 127 frames for the existing $4-\mathrm{Mb} / \mathrm{s}$ links in an effort to solve the existing problem. Fig. 4 also plots throughput efficiency versus link BER for 4-Mb/s links with $t_{\mathrm{ta}}=10 \mathrm{~ms}, l=16 \mathrm{~kb}$, and $W_{\max }=127$ frames. Throughput efficiency significantly increases with the 127 window-size employment and reaches the acceptable value of 0.96 . Fig. 5 plots throughput efficiency versus link BER. It examines the effect of reducing $t_{\mathrm{ta}}$ and/or increasing window size in throughput efficiency for a $16-\mathrm{Mb} / \mathrm{s}$ link. Throughput efficiency for $t_{\mathrm{ta}}=10 \mathrm{~ms}$ and $W_{\max }=7$ shows that the increased turnaround frequency results in poor performance. Reducing acknowledgement time portion by only increasing window size $\left(t_{\mathrm{ta}}=10 \mathrm{~ms}\right.$ and $\left.W_{\max }=127\right)$ results in a significant increase but yet a questionable performance. By reducing only $t_{\mathrm{ta}}\left(t_{\mathrm{ta}}=0.1 \mathrm{~ms}\right.$ and $\left.W_{\max }=7\right)$, an excellent performance is observed. Taking further advantage of the optional window size increase $\left(t_{\mathrm{ta}}=0.1 \mathrm{~ms}\right.$ and $\left.W_{\max }=127\right)$ results in a slightly better performance for low BER but renders the link vulnerable to BER increase as it requires a link BER of $10^{-8}$ to achieve an excellent performance as opposed to a $10^{-7} \mathrm{BER}$ requirement for $W_{\max }=7$. As a conclusion, $t_{\mathrm{ta}}$ adjustment is a necessity while the effectiveness of window size increase is debatable. 


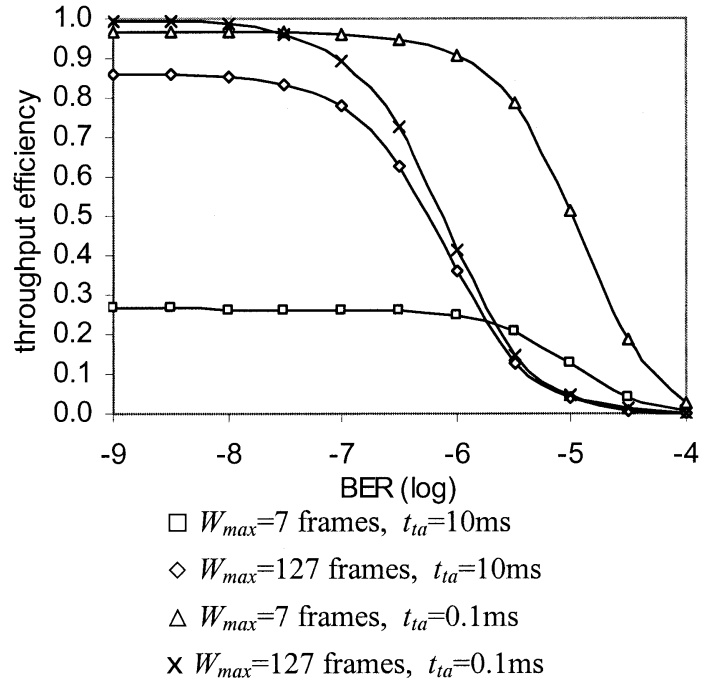

Fig. 5. Throughput efficiency versus BER for $C=16 \mathrm{Mb} / \mathrm{s}, l=16 \mathrm{~kb}$, and $t_{\mathrm{Fout}}=t_{\mathrm{Imax}}+2 t_{\mathrm{ta}}$.

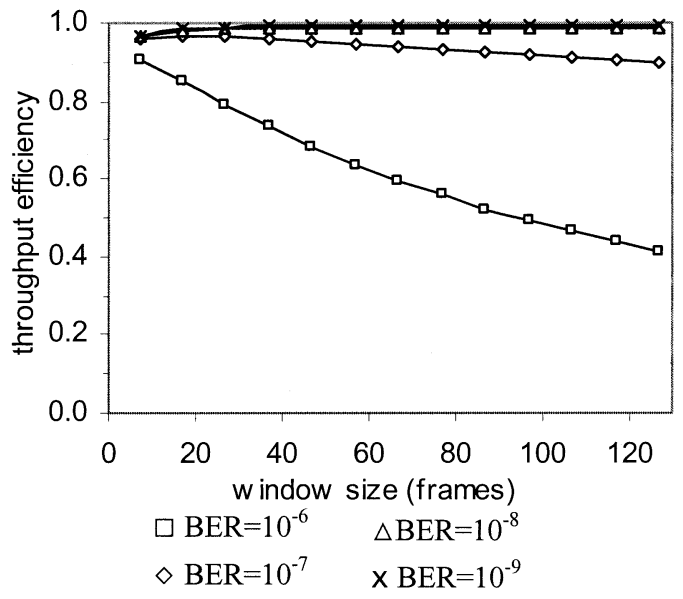

Fig. 6. Throughput efficiency versus window size for $C=16 \mathrm{Mb} / \mathrm{s}, t_{\mathrm{ta}}=$ $0.1 \mathrm{~ms}, l=16 \mathrm{~kb}$, and $t_{\mathrm{Fout}}=t_{\mathrm{Imax}}+2 t_{\mathrm{ta}}$.

Fig. 6 plots throughput efficiency versus window size for different link BERs for 16-Mb/s links. Window size increase results in slight throughput increase for low BERs and significant decrease for high BERs. Fig. 7 plots throughput efficiency versus window size for $4-\mathrm{Mb} / \mathrm{s}$ links with $t_{\mathrm{ta}}=10 \mathrm{~ms}$. A much different behavior is observed due to the large link turnaround time value as related to link data rate. A significant throughput increase with window size increase for low BER is observed as the link turnaround frequency is decreased. This also applies for high BER $\left(10^{-6}\right)$ but when window size becomes very large, a throughput decrease is observed caused by the increased number of retransmitted frames following an error frame in a window transmission.

Fig. 8 shows the percent time consumed for different IrLAP tasks for the $16-\mathrm{Mb} / \mathrm{s}$ link with $W_{\max }=127$ and $t_{\mathrm{ta}}=0.1 \mathrm{~ms}$. It reveals that for large window-size values $\left(W_{\max }=127\right)$, the key factor that reduces throughput for a wide range of BER (from $10^{-8}$ to $10^{-4}$ ) is the retransmission of correctly received out-of-sequence $I$ frames. This is a limitation of the IrDA IrLAP protocol when nonoptimum window size is used,

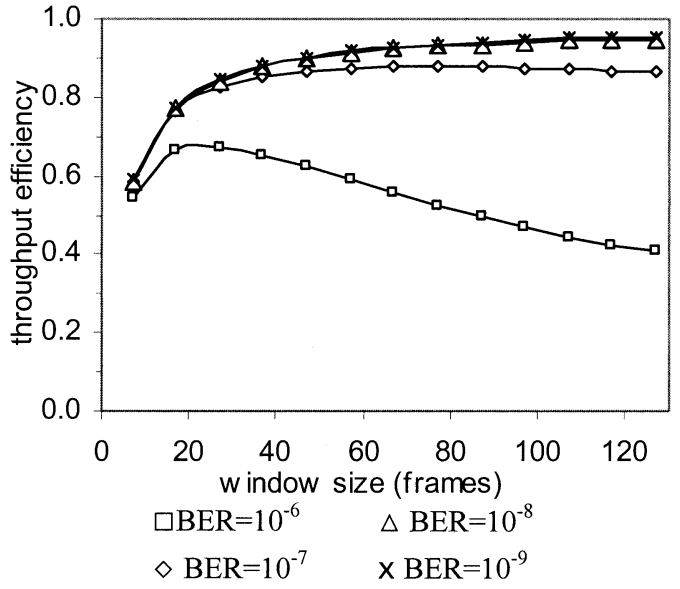

Fig. 7. Throughput efficiency versus window size for $C=4 \mathrm{Mb} / \mathrm{s}, t_{\mathrm{ta}}=$ $10 \mathrm{~ms}, l=16 \mathrm{~kb}$, and $t_{\mathrm{Fout}}=t_{\mathrm{Imax}}+2 t_{\mathrm{ta}}$.

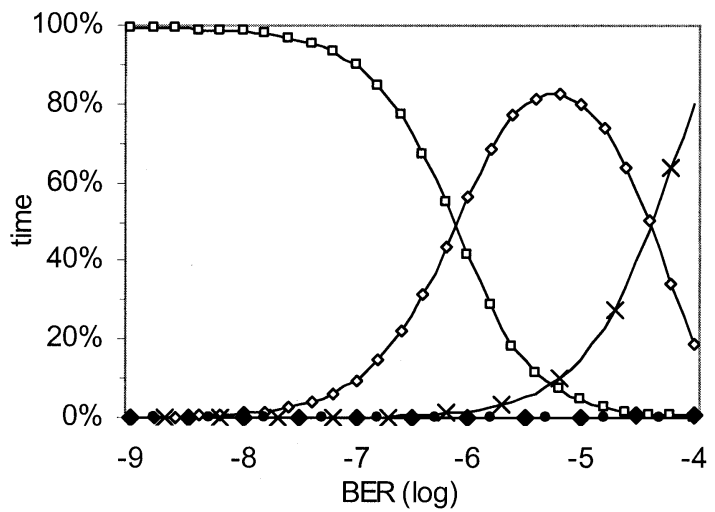

useful data transmission (throughput efficiency)

retransmission of correctly received out of sequence frames

retransmission of error frames

F-timer expiration

reversing link direction (hardware latency)

Fig. 8. Time allocation of various IrLAP tasks against BER for $C=16 \mathrm{Mb} / \mathrm{s}$, $l=16 \mathrm{~kb}, t_{\mathrm{ta}}=0.1 \mathrm{~ms}, W_{\max }=127$ frames, and $t_{\mathrm{Fout}}=t_{\mathrm{Imax}}+2 t_{\mathrm{ta}}$.

especially for high BER. Fig. 9 plots throughput efficiency versus frame size for $16-\mathrm{Mb} / \mathrm{s}$ links with $t_{\mathrm{ta}}=0.1 \mathrm{~ms}$ and $W_{\max }=127$. It shows that, although at low BER the maximum frame size should be used, a much different frame-size value should be used at high BER for maximum throughput. Thus, optimum window and frame-size parameters are of great importance for IrLAP throughput.

\section{OPTIMUM LinK PARAMETER VALUES}

Throughput can be maximized by using optimum window and frame-size values for the link BER. Optimum values are derived by setting the first derivative of (15) equal to zero. Throughput analysis presented in the previous section revealed the importance of minimum turnaround time, which is a physical layer parameter. Optimum $t_{\text {Fout }}$ value must first be examined for maximum throughput at high BER.

Equation (5) shows that if the link BER $p_{b}$ is increased, frame error probability $p$ is significantly increased. In such a case, the time spent on primary's $F$-timer expiration, represented in (9) 


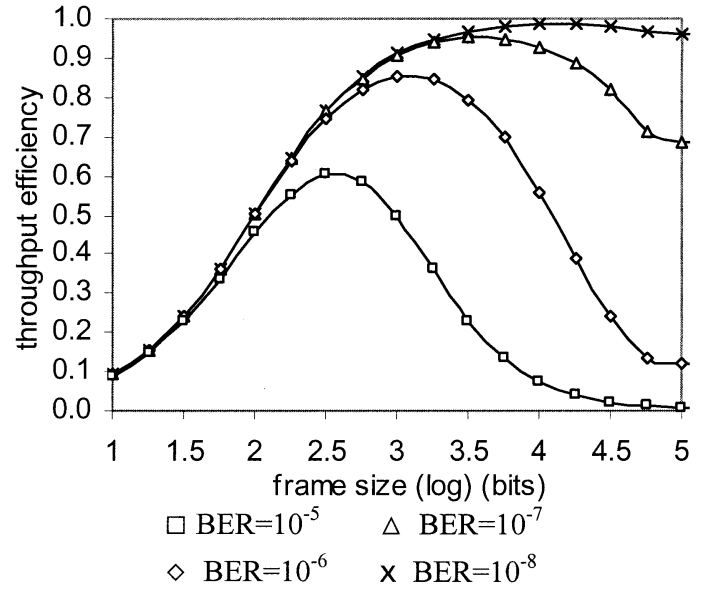

Fig. 9. Throughput efficiency versus frame size for $C=16 \mathrm{Mb} / \mathrm{s}, t_{\mathrm{ta}}=$ $0.1 \mathrm{~ms}, W_{\max }=127$ frames, and $t_{\text {Fout }}=t_{\mathrm{Imax}}+2 t_{\mathrm{ta}}$.

by term $p t_{\text {Fout }}$, may significantly increase the average WTT resulting in throughput performance degradation. IrLAP specification [9] poses only an upper limit of $500 \mathrm{~ms}$ for the $t_{\text {Fout }}$ value and allows the implementation of smaller values. According to IrLAP specification [9], if the secondary has information for transmission, it sets the $F$ bit in the last $I$ frame it transmits. Otherwise, upon gaining transmission control, it immediately transmits an $S$ frame with the $F$ bit set, thus, acknowledging correctly received $I$ frames and reversing link direction. Thus, the secondary station never holds transmission control without transmitting $I$ frames. As a result, the $t_{\text {Fout }}$ value may be safely reduced from the value of $500 \mathrm{~ms}$ to the smaller time period required for the secondary to transmit a full window $(N)$ of full payload $(l=16 \mathrm{~kb}) I$ frames plus the time required for reversing the link direction twice, $t_{\text {Fout }}=N t_{\text {Imax }}+2 t_{\text {ta. }}$. This $t_{\text {Fout }}$ value assumes that the secondary has transmitted a full window of $I$ frames and the primary did not manage to correctly receive a single $I$ frame. In the saturation case considered in this work, the secondary station never transmits $I$ frames to the primary and immediately acknowledges correctly received $I$ frames by means of an $S$-frame transmission. As a result, a smaller $t_{\text {Fout }}$ value of $t_{\text {Fout }}=t_{\text {Imax }}+2 t_{\text {ta }}$ may be safely implemented in the current scenario. This value allows the secondary station to transmit an $S$ frame or an $I$ frame if it wishes to transmit information data at the end of information transfer from the primary to the secondary station. This $t_{\text {Fout }}$ value is valid since it corresponds to a maximum window-size parameter of one for the primary station negotiated and agreed during link establishment.

The $t_{\text {Fout }}$ value becomes of key importance for maximum throughput at high BER if optimum link-layer parameter values are implemented by the primary station. Fig. 10 shows the time allocation of various IrLAP tasks versus BER when optimum window size $N$ is implemented for $t_{\text {Fout }}=500 \mathrm{~ms}$. At high BER, a significant amount of time is spent on $F$-timer expiration causing serious throughput degradation. It can be easily observed that the time portion utilized on $F$-timer expiration is much larger than the time portion utilized on other IrLAP tasks, such as retransmitting error frames, retransmitting correctly received out-of-sequence frames, and reversing link di-

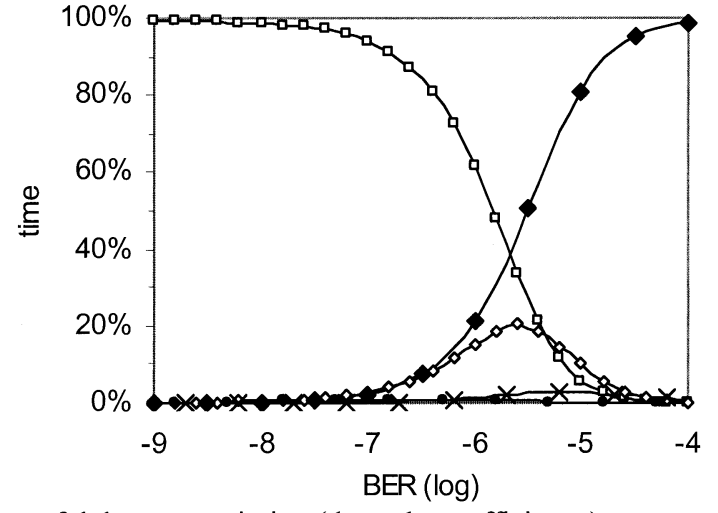

$\square$ useful data transmission (throughput efficiency)

$\diamond$ retransmission of correctly received out of sequence frames

$X$ retransmission of error frames

- F-timer expiration

- reversing link direction (hardware latency)

Fig. 10. Time allocation of various IrLAP tasks against BER for $N$ optimum, $t_{\text {Fout }}=500 \mathrm{~ms}, C=16 \mathrm{Mb} / \mathrm{s}, l=16 \mathrm{~kb}$, and $t_{\mathrm{ta}}=0.1 \mathrm{~ms}$.

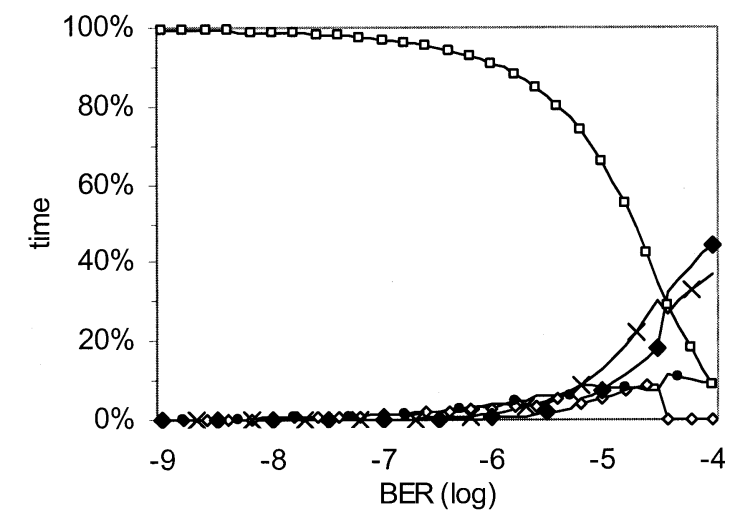

$\square$ useful data transmission (throughput efficiency)

$\diamond$ retransmission of correctly received out of sequence frames

$X$ retransmission of error frames

- F-timer expiration

- reversing link direction (hardware latency)

Fig. 11. Time allocation of various IrLAP tasks against BER for $N$ optimum, $t_{\mathrm{Fout}}=t_{\mathrm{Imax}}+2 t_{\mathrm{ta}}, C=16 \mathrm{Mb} / \mathrm{s}, l=16 \mathrm{~kb}$, and $t_{\mathrm{ta}}=0.1 \mathrm{~ms}$.

rection. The situation is explained by considering that a single $I$-frame transmission error results in a significant 500 -ms delay if the lost $I$ frame contains the $P$ bit. For the saturation case considered, if the maximum window size allowed for the primary is agreed to be equal to one and $t_{\text {Fout }}=t_{\text {Imax }}+2 t_{\text {ta }}$, a much different behavior is shown in Fig. 11. A significant throughput improvement is achieved over a wide BER range (from $10^{-7}$ to $10^{-4}$ ) mainly by taking advantage of time otherwise wasted on $F$-timer expiration. Unless otherwise specified, the $t_{\text {Fout }}$ value implemented in this work is given by

$$
t_{\text {Fout }}=t_{\text {Imax }}+2 t_{\text {ta }} \text {. }
$$

A different approach to address the significant delays arising from the $F$-timer expiration is to reduce the probability of $P$ bit loss rather than reducing the time wasted for every $P$ bit loss. 
According to the IrLAP specification state charts [9], the primary station sets the $P$ bit in the last $I$ frame in a window transmission. This decision assumes that link BER is very low and frame error probability $p$ is very small. Thus, the $P$ bit is seldom lost and time spent on $F$-timer expiration is negligible. However, if link BER is relatively high, $p$ is significantly increased as it usually refers to an $I$ frame with $16 \mathrm{~kb}$ of user data. To reduce the probability of $P$ bit loss, an IrLAP modification may be employed. The primary should not set the $P$ bit in the last $I$ frame it transmits, but transmit the $P$ bit in a new RR $S$ frame that follows the last $I$-frame transmission. As $S$ frames are very small, the new RR frames introduce negligible additional delays. As $S$ frames have very small frame error rates, delays on $F$-timer expiration are significantly reduced. The mathematical model presented in this work can be easily altered to calculate throughput performance for the proposed IrLAP improvement. $S$-frame modification is presented in Fig. 3(d) and (e) and WTT becomes

$$
t_{w-\mathrm{RR}}=N t_{I}+t_{s}+t_{\mathrm{ack}}
$$

independent of the number of $I$-frame errors in the window transmission. The assumption that $S$ frames are always transmitted error free holds true since the $S$-frame error rate is well below 0.01 at the highest BER value considered in this work. Throughput is now given by

$$
D_{b-\mathrm{RR}}=l \frac{1-p}{p} \frac{\left(1-(1-p)^{N}\right)}{N t_{I}+t_{s}+t_{\mathrm{ack}}} .
$$

The following analysis for deriving optimum values for window and/or frame-size parameters does not consider links employing the $S$-frame modification and using small $t_{\text {Fout }}$ values, such as the value given in (22). Identical formulas have been derived for the $S$-frame modification (which eliminates $F$-timer delays) by taking the first derivative of (24). Hence, the following results and conclusions apply to both cases.

\section{A. Optimum Window Size}

Due to the half duplex operation of the IrLAP protocol, window size is a very important and easily adjustable parameter. If a small window-size value is implemented, the increased link turnaround frequency results in significant delays and decreases throughput performance. If a high window-size value is implemented at high BER, a large number of frames following an error frame may be transmitted. Even if these frames are correctly received, they are considered as out of sequence and are discarded by the receiver. These frame transmissions essentially delay reversing link direction, acknowledging correctly received frames, and retransmitting the erred frame.

To derive optimum window-size values, the derivative of (15) against $N$ must be set to zero. First, considering the valid approximation for small $p$

$$
(1-p)^{N} \approx 1-N p+\frac{N(N-1)}{2} p^{2}
$$

the derivative of (15) becomes

$$
\frac{\partial D_{b}}{\partial N}=\frac{l}{p+1} \frac{1-p}{2} \frac{\partial}{\partial N} \frac{2 N-N(N-1) p}{N t_{I}+\frac{2 p t_{\mathrm{ta}}+p t_{s}+t_{\mathrm{ack}}}{p+1}}=0 .
$$

After some algebra and assuming $2 p t_{\mathrm{ta}}+p t_{s}+t_{\mathrm{ack}} /(p+1) \approx$ $t_{\mathrm{ack}}$

$$
\left(-p t_{I}\right) N^{2}+\left(-2 p t_{\text {ack }}\right) N+2 t_{\text {ack }}+p t_{\text {ack }}=0 .
$$

Assuming $p t_{\text {ack }} \ll 2 t_{\text {ack }}$ and $-2 p t_{\text {ack }}<-p t_{I}$, (27) becomes

$$
\left(-p t_{I}\right) N^{2}+2 t_{\text {ack }}=0
$$

and

$$
N_{\mathrm{opt}}=\sqrt{\frac{2 t_{\mathrm{ack}}}{p t_{I}}} .
$$

Considering the valid approximations for small $p_{b}$ and $l \gg l^{\prime}$, $p \approx l p_{b}$ and $t_{I} \approx l / C$, optimum window-size value is given by

$$
N_{\text {opt }}=\sqrt{\frac{2 t_{\mathrm{ack}} C}{l^{2} p_{b}}} .
$$

The optimum window-size values versus BER for fixed frame size are shown in Fig. 12. Window size should be decreased with the increase of BER for maximum throughput. Note that at very low BER, the optimum window-size values should be greater than the maximum window-size value of 127 frames allowed by the IrLAP specification. Fig. 12 also compares $N_{\text {opt }}$ values derived from (30) with optimum window-size values obtained using exact numerical methods in (15) for a $16-\mathrm{Mb} / \mathrm{s}$ link with $t_{\mathrm{ta}}=0.1 \mathrm{~ms}$. An exact match is observed validating the approximations used to derive (30).

\section{B. Optimum Frame Size}

A different approach for reducing information transmitted in a window transmission is by decreasing frame size. A small frame size reduces frame error probability and the necessity for retransmissions. However, as each frame transmission requires the transmission of flags, address field, control field, and FCS, employing small frame sizes results in a relative increase of overheads. In addition, frame-size adjustment requires buffer reorganization if adjustment on frame retransmissions is implemented. Thus, optimum frame-size implementation is more difficult than optimum window-size implementation but it may also be employed for increasing throughput performance.

The following approximations are considered for small $p_{b}$

$$
\begin{aligned}
p & =1-\left(1-p_{b}\right)^{l+l^{\prime}} \approx\left(l+l^{\prime}\right) p_{b} \\
\frac{1-p}{p} & =\frac{1}{p}-1 \approx \frac{1}{p} \\
\left(l+l^{\prime}\right) p_{b} t_{\mathrm{Fout}} & =0 \\
\left(l+l^{\prime}\right) p_{b} t_{s} & =0 \\
1-\left(1-\left(l+l^{\prime}\right) p_{b}\right)^{N} & \approx N\left(l+l^{\prime}\right) p_{b}-\frac{N(N-1)}{2}\left(l+l^{\prime}\right)^{2} p_{b}^{2}
\end{aligned}
$$

and $D_{b}$ is given, to a good approximation, by

$$
D_{b}=\frac{C}{2 N p_{b}} l \frac{2 N p_{b}-N(N-1)\left(l+l^{\prime}\right) p_{b}^{2}}{\left(l+l^{\prime}\right)+\frac{t_{\text {ack }} C}{N}} .
$$




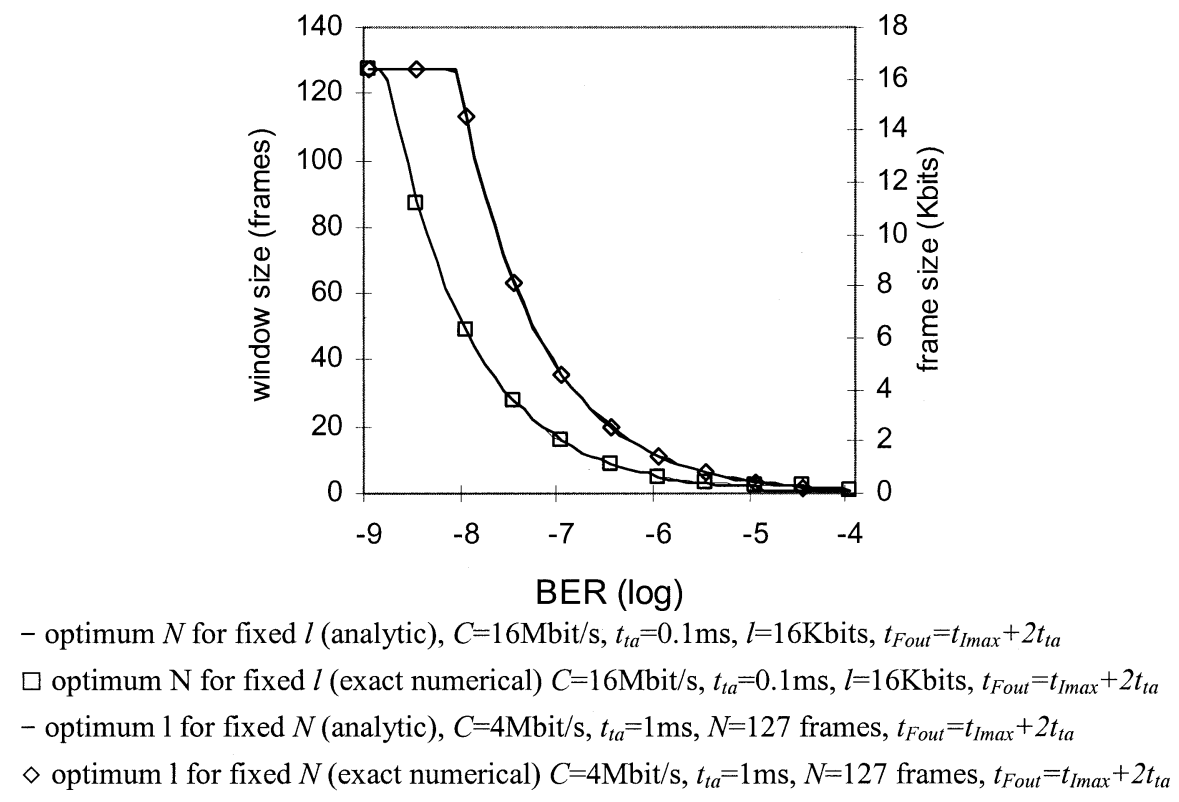

Fig. 12. Optimum window/frame-size validation.

The derivative against $l$ is taken, set equal to zero, and after some algebra, we derive that optimum frame-size values are given to a good approximation by

$$
l_{\mathrm{opt}}=\sqrt{\frac{2\left(N l^{\prime}+t_{\mathrm{ack}} C\right)}{N^{2} p_{b}}} .
$$

The optimum frame-size values versus BER for a fixed window size of 127 are shown in Fig. 12. As expected, frame size should be decreased at high BER if maximum throughput is to be achieved. Note that at very low BER, the optimum frame-size values should be greater than the maximum frame-size value of $16 \mathrm{~kb}$ allowed by the IrLAP specification. As in the case of optimum window-size values, all approximations considered in deriving (37) are validated by comparing optimum values given from (37) with optimum values derived by employing numerical methods in (15) for a $4-\mathrm{Mb} / \mathrm{s}$ link with $t_{\mathrm{ta}}=1 \mathrm{~ms}$.

An important conclusion can be extracted by observing that (30) and (37) that calculate optimum values can be rewritten as

$$
N l p_{b} \frac{N l}{2}=t_{\mathrm{ack}} C+N l^{\prime}
$$

Equation (38) reveals that maximum throughput is achieved when the probability of a bit error in the optimum window frame transmission $\left(\approx N l p_{b}\right)$ times the number of bits that have to be retransmitted due to this error, which on average is half the window transmission $N l / 2$, is equal to the acknowledgement time in bits $t_{\mathrm{ack}} C$ plus the number of overhead bits in the window transmission $N l^{\prime}$.

The term $\mathrm{Nl}^{\prime}$ is missing from (30) because, if optimum window-size values are implemented, optimum $N$ becomes relatively small for high BERs, so term $N l^{\prime}$ can safely be neglected.

\section{Simultaneous Optimization of Window and Frame Size}

If window- and frame-size link parameters can be simultaneously adjusted, maximum throughput performance can be achieved. Optimum $N$ and $l$ values are derived by taking $\partial D_{b} / \partial N=\partial D_{b} / \partial l=0$. First, the derivative versus $N$ can be taken following the analysis in Section VI-A. Optimum $N$ values derived by setting the derivative equal to zero can be substituted to throughput equation. Throughput $D_{b}$ is now a function of frame size $l$ for optimum $N$ values. The derivative versus $l$ can now be taken and set equal to zero to derive optimum $l$ values. $N_{\text {opt }}$ given by (29) should be used as the assumption $l \gg l^{\prime}$ is no longer valid as optimum $l$ values may be significantly small.

$$
N_{\mathrm{opt}}=\sqrt{\frac{2 t_{\mathrm{ack}}}{p t_{I}}} \approx \sqrt{\frac{2 t_{\mathrm{ack}}}{\left(l+l^{\prime}\right) p_{b} \frac{l+l^{\prime}}{C}}}=\sqrt{\frac{2 t_{\mathrm{ack}} C}{\left(l+l^{\prime}\right)^{2} p_{b}}}
$$

and

$$
N_{\text {opt }}\left(l+l^{\prime}\right)=\sqrt{\frac{2 t_{\mathrm{ack}} C}{p_{b}}}=c .
$$

Considering (5), throughput (15) can be rewritten as

$$
D_{b}=\frac{l(1-p)}{p} \frac{\left(1-\left(1-p_{b}\right)^{N\left(l+l^{\prime}\right)}\right)}{N \frac{l+l^{\prime}}{C}+p\left(t_{\mathrm{Fout}}+t_{s}\right)+t_{\text {ack }}} .
$$

Considering (5) and (40)

$$
D_{b}=\frac{l(1-p)}{p} \frac{\left(1-\left(1-p_{b}\right)^{c}\right)}{\left(\frac{c}{C}+p\left(t_{\text {Fout }}+t_{s}\right)+t_{\text {ack }}\right)} .
$$

Assuming the valid approximation $p \approx\left(l+l^{\prime}\right) p_{b}$, then

$$
\begin{aligned}
D_{b}= & \frac{\left(1-\left(1-p_{b}\right)^{c}\right)}{\frac{c}{C}} \\
& \times \frac{l\left(1-\left(l+l^{\prime}\right) p_{b}\right)}{\left(l+l^{\prime}\right) p_{b}\left(1+\left(l+l^{\prime}\right) p_{b} \frac{\left(t_{\text {Fout }}+t_{s}\right) C}{c}+\frac{t_{\text {ack }} C}{c}\right)} .
\end{aligned}
$$


Denoting $s=\left(t_{\text {Fout }}+t_{s}\right) C / c$, taking the first derivative versus $l$ and by setting it to zero

$$
\begin{aligned}
& \left(-2 l p_{b}-\left(l^{\prime} p_{b}-1\right)\right)\left(\left(l+l^{\prime}\right)^{2} p_{b}^{2} s+\left(l+l^{\prime}\right) p_{b}\left(1+\frac{t_{\mathrm{ack}} C}{c}\right)\right) \\
& +\left(l^{2} p_{b}+l\left(l^{\prime} p_{b}-1\right)\right)\left(2\left(l+l^{\prime}\right) p_{b}^{2} s+p_{b}\left(1+\frac{t_{\mathrm{ack}} C}{c}\right)\right)=0 .
\end{aligned}
$$

Considering the valid approximation $l^{\prime} p_{b} \approx 0$, we reach, after some algebra

$$
\begin{gathered}
-l^{2} p_{b}\left(s+2 l^{\prime} p_{b} s+1+\frac{t_{\mathrm{ack}} C}{c}\right) \\
+p_{b} l\left(-2 l^{\prime 2} p_{b} s-2 l^{\prime}\left(1+\frac{t_{\mathrm{ack}} C}{c}\right)\right) \\
+l^{\prime 2} p_{b} s+l^{\prime}\left(1+\frac{t_{\mathrm{ack}} C}{c}\right)=0
\end{gathered}
$$

As $-2 l^{\prime 2} p_{b} s-2 l^{\prime}\left(1+t_{\text {ack }} C / c\right) \approx 0$

$$
l_{\mathrm{opt}}^{2}=\frac{l^{\prime}\left(l^{\prime} p_{b} s+1+\frac{t_{\mathrm{ack}} C}{c}\right)}{p_{b}\left(s+2 l^{\prime} p_{b} s+1+\frac{t_{\mathrm{ack}} C}{c}\right)}
$$

and, to a very good approximation

$$
l_{\mathrm{opt}} \approx \sqrt{\frac{l^{\prime}}{p_{b}}}
$$

By substituting (47) into (40), we obtain

$$
N_{\mathrm{opt}} \approx \sqrt{\frac{2 t_{\mathrm{ack}} C}{l^{\prime}}} .
$$

Fig. 13 plots simultaneously optima window and frame-size values for $4-\mathrm{Mb} / \mathrm{s}$ links with $t_{\mathrm{ta}}=0.1 \mathrm{~ms}$. It is observed that for a high range of BERs (less than $10^{-6.5}$ ), (47) suggests that frame-size values greater than $16 \mathrm{~kb}$ (the maximum allowed for IrLAP) should be employed. For this range, optimum $N$ values are given by (30) instead of (48) since frame-size values are constant $(l=16 \mathrm{~kb})$ and not optimum. As a very good match between values given by (30), (47), and (48) and optimum values derived by using numerical methods is observed, approximations made to derive (47) and (48) are validated. Slight differences are observed mainly for high BER because optimum $N$ values given by the mathematical analysis and (48) are real values and have to be rounded as $N$ can, of course, take only integer values.

An important conclusion can be extracted by observing that (30) and (47) for optimum values can be rewritten as

$$
\begin{aligned}
N_{\mathrm{opt}} l_{\mathrm{opt}} p_{b} \frac{N_{\mathrm{opt}} l_{\mathrm{opt}}}{2} & =t_{\mathrm{ack}} C \\
l_{\mathrm{opt}} l_{\mathrm{opt}} p_{b} & =l^{\prime} .
\end{aligned}
$$

Equation (50) reveals that optimum throughput is achieved when the probability of a bit error in the optimum frame $(\approx$ $\left.l_{\text {opt }} p_{b}\right)$ times the number of bits that have to be retransmitted due to this error $\left(\approx l_{\text {opt }}\right)$ must be equal to the frame bit overhead $l^{\prime}$. This equation shows that optimum frame-size values should balance between time spent on retransmitting error frames and time spent on transmitting overheads. Equation (49) shows that maximum throughput is achieved when the probability of a bit error

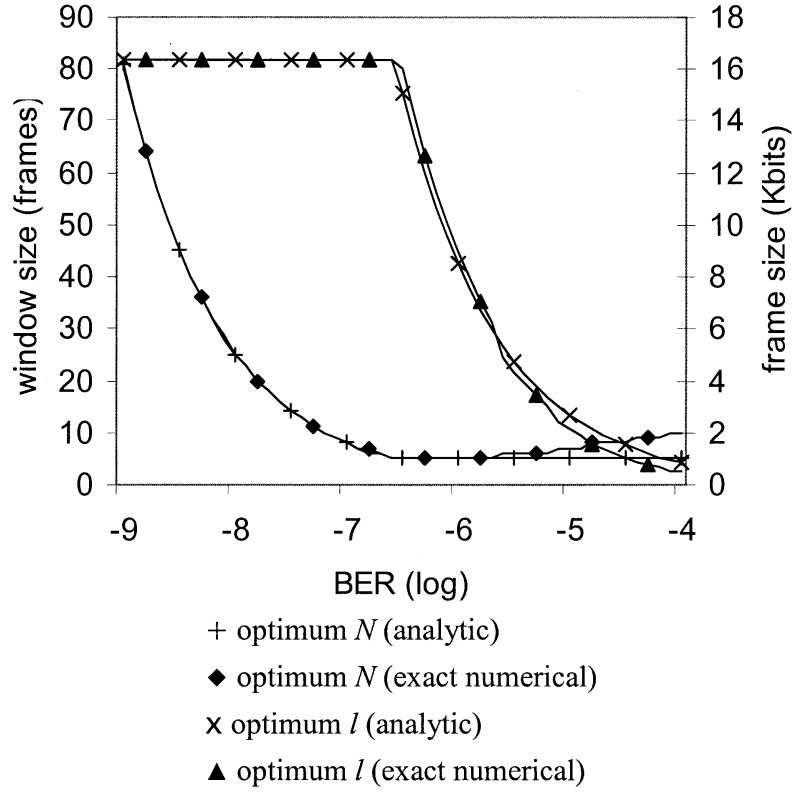

Fig. 13. Simultaneously optima window and frame-size validation for $C=$ $4 \mathrm{Mb} / \mathrm{s}, t_{\mathrm{ta}}=0.1 \mathrm{~ms}$, and $t_{\mathrm{Fout}}=t_{\text {Imax }}+2 t_{\mathrm{ta}}$.

in the optimum window frame transmission $\left(\approx N_{\text {opt }} l_{\text {opt }} p_{b}\right)$ times the number of bits that have to be retransmitted in the following frames due to this error, which on average is half the window transmission $N_{\text {opt }} l_{\text {opt }} / 2$, is equal to the acknowledgement time in bits $t_{\text {ack }} C$. In other words, the bits transmitted in the optimum window transmission $N_{\text {opt }} l_{\text {opt }}$ should balance the time utilized in retransmitting out-of-sequence frames and time utilized on acknowledgements.

\section{Throughput ANALYsis USING Optimum VAlueS}

Fig. 14 compares the throughput efficiency of a 16-Mb/s link with $t_{\mathrm{ta}}=0.1 \mathrm{~ms}$ employing $N=127$ and $l=16 \mathrm{~kb}$ with the throughput achieved by implementing optimum window-size or frame-size values given by (30) and (37), respectively. It shows that throughput is significantly increased for a wide range of BER values (from $10^{-7}$ to $10^{-4}$ ) if optimum window or frame-size values are employed. For low BER, throughput performance for optimum $N$ values is higher than throughput performance for optimum $l$ values because as window size decreases, fewer frame overheads $l^{\prime}$ are utilized in a window transmission. The situation is reversed for high BER because the optimum window-size implementation always utilizes large frame sizes $(l=16 \mathrm{~kb})$, has a high frame error probability at high BER, and often the $P$ bit is lost. Implementing the suggested modification in Section VI and setting the $P$ bit in a special RR $S$ frame is always beneficial as it eliminates time spent on $F$ timer. Fig. 14 also shows that applying optimum window and frame-size values simultaneously results in better performance overall.

Fig. 15 shows the effect of reducing $t_{\mathrm{ta}}$ to $0.01 \mathrm{~ms}$ for the same link parameters as in Fig. 14. A throughput increase is observed, especially for the simultaneous implementation of optimum window and frame-size values. This is due to the increased link turnaround frequency caused by the employment of optimum (smaller) window and frame-size values. As a 


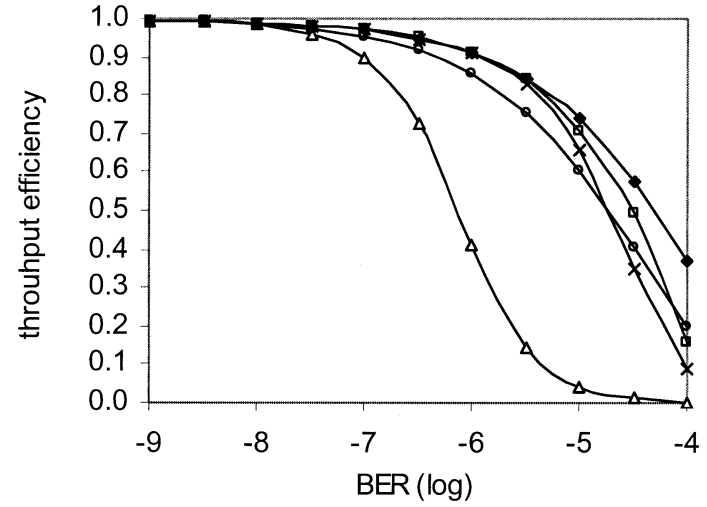

$\Delta N=127$ frames, $l=16 \mathrm{Kbits}, t_{\text {Fout }}=t_{\text {Imax }}+2 t_{\text {ta }}$

$\mathrm{x}$ optimum $N, l=16 \mathrm{Kbits}, t_{\text {Fout }}=t_{\text {Imax }}+2 t_{\text {ta }}$

$\circ$ optimum $l, N=127$ frames, $t_{\text {Fout }}=t_{\text {Imax }}+2 t_{\text {ta }}$

$\square$ optimum $N$, P bit in RR-frame, $l=16 \mathrm{Kbits}$

• optimum $N$ and optimum $l, t_{\text {Fout }}=t_{\text {Imax }}+2 t_{t a}$

Fig. 14. Throughput efficiency against BER for $C=16 \mathrm{Mb} / \mathrm{s}$ and $t_{\mathrm{ta}}=$ $0.1 \mathrm{~ms}$.

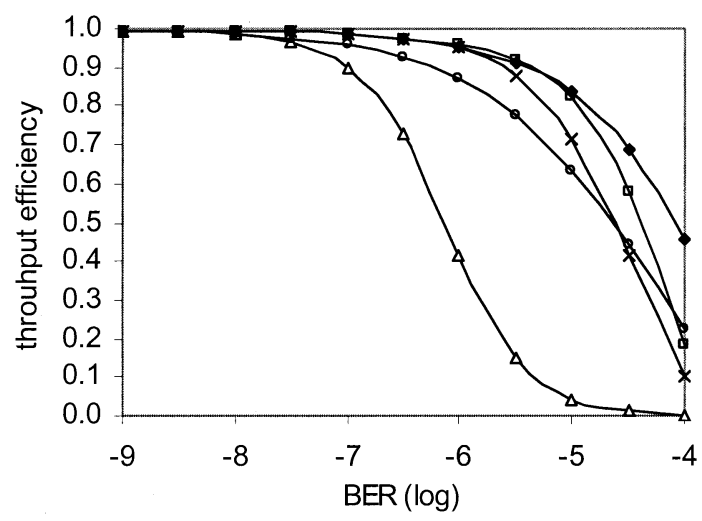

$\Delta N=127$ frames, $l=16 \mathrm{Kbits}, t_{\text {Fout }}=t_{\text {Imax }}+2 t_{\text {ta }}$

$\mathrm{x}$ optimum $N, l=16 \mathrm{Kbits}, t_{\text {Fout }}=t_{\text {Imax }}+2 t_{\text {ta }}$

o optimum $l, N=127$ frames, $t_{\text {Fout }}=t_{\text {Imax }}+2 t_{\text {ta }}$

$\square$ optimum $N$, P bit in RR-frame, $l=16 \mathrm{Kbits}$

- optimum $N$ and optimum $l, t_{\text {Fout }}=t_{\text {Imax }}+2 t_{\text {ta }}$

Fig. 15. Throughput efficiency against BER for $C=16 \mathrm{Mb} / \mathrm{s}$ and $t_{\mathrm{ta}}=$ $0.01 \mathrm{~ms}$.

result, for comparison, achieving the same high throughput is possible with a link of $10^{-5}$ BER using the simultaneous window and frame-size implementation as opposed to a $10^{-7}$ BER required for $N=127$ frames and $l=16 \mathrm{~kb}$. Fig. 16 shows the percent time portion utilized for various tasks for the same link parameters and reveals that the retransmission of correctly received out-of-sequence frames is of no importance any more as optimum window and frame-size employment reduced the probability of transmitting out-of-sequence frames. It also shows that the $F$-timer expiration becomes again of great importance at very high BER. If the $P$ bit is transmitted on a special $S$ frame, as suggested in Section VI, a significant increase in throughput efficiency is observed in Fig. 17. Links employing a small $t_{\mathrm{ta}}$ of $0.01 \mathrm{~ms}$ and implementing the proposed $S$-frame modification, a throughput efficiency of $65 \%$ can be achieved

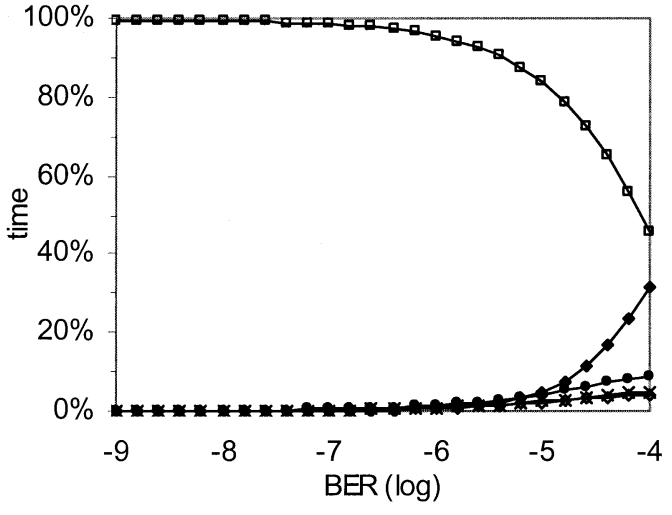

$\square$ useful data transmission (throughput efficiency)

$\diamond$ retransmission of correctly received out of sequence frames

$X$ retransmission of error frames

- F-timer expiration

- reversing link direction (hardware latency)

Fig. 16. Time allocation of various IrLAP tasks against BER for simultaneous optima $N$ and $l, C=16 \mathrm{Mb} / \mathrm{s}, t_{\mathrm{ta}}=0.01 \mathrm{~ms}$, and $t_{\mathrm{Fout}}=t_{\mathrm{Imax}}+2 t_{\mathrm{ta}}$

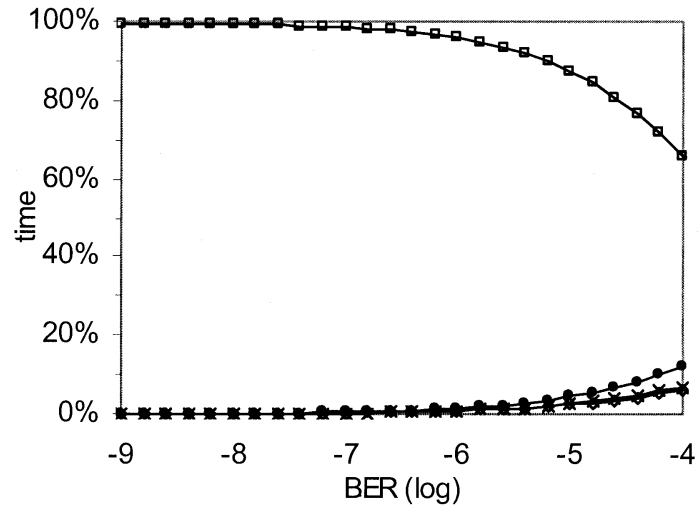

$\square$ useful data transmission (throughput efficiency)

$\diamond$ retransmission of correctly received out of sequence frames

$X$ retransmission of error frames

- reversing link direction (hardware latency)

Fig. 17. Time allocation of various IrLAP tasks against BER for simultaneous optima $N$ and $l, P$ bit in $S$ frame, $C=16 \mathrm{Mb} / \mathrm{s}$ and $t_{\mathrm{ta}}=0.01 \mathrm{~ms}$.

even for a $10^{-4}$ BER by employing optimum widow and frame-size values.

\section{CONCLUSION}

A mathematical model based on the average WTT has been developed to study the IrLAP throughput performance. The model leads to simple formulas for IrLAP link-layer throughput and illuminates on the time-consuming tasks and delays involved in IrLAP operation. The derived formulas relate throughput and delays with parameters such as link BER, link data rate, minimum turnaround time, frame size, and window size. Throughput results are presented for different link parameters. The significance of the minimum turnaround time delay on throughput is revealed and explored for different IrDA links. Small minimum turnaround delays should be implemented if maximum throughput is to be achieved. The 
effectiveness of the proposed larger window size of 127 frames for the $16-\mathrm{Mb} / \mathrm{s}$ links becomes questionable as it slightly increases throughput at low BER but renders link operation very vulnerable to BER increase. The importance of $F$-timer value is also explored at high BER.

Mathematical analysis allows us to derive optimum window and frame-size values for any BER. Results indicate that throughput performance is highly improved by simultaneous optimum window and frame-size employment. This suggests that adaptive algorithms for modifying window size and frame length would be beneficial at high BER. A protocol improvement that utilizes special RR frames to pass transmission control is proposed. Special RR frame employment eliminates delays due to $F$-timer expiration and significantly improves link-layer throughput when optimum window and frame-size values are simultaneously employed. The analytical results and optimum values derived can be very useful to link designers in determining the effectiveness of physical and link-layer parameters in IrDA link performance.

\section{REFERENCES}

[1] D. J. T. Heatly, D. R. Wisely, I. Neild, and P. Cochrane, "Optical wireless: The story so far," IEEE Commun. Mag., vol. 36, pp. 72-82, Dec. 1998.

[2] A. C. Boucouvalas and Z. Ghassemlooy, "Editorial, special issue on optical wireless communications," in Proc. Inst. Elect. Eng. J, Optoelectron., vol. 147, 2000, p. 279.

[3] S. Williams, "IrDA: Past, present and future," IEEE Pers. Commun., vol. 7, pp. 11-19, Feb. 2000.

[4] I. Millar, M. Beale, B. J. Donoghue, K. W. Lindstrom, and S. Williams, "The IrDA standard for high-speed infrared communications," HewlettPackard J., vol. 49, no. 1, pp. 10-26, 1998.

[5] IrDA, Serial Infrared Physical Layer Specification - Version 1.4, Infrared Data Association, 2001.

[6] IrDA, Serial Infrared Physical Layer Specification - Version 1.0, Infrared Data Association, 1994.

[7] IrDA, Serial Infrared Physical Layer Specification - Version 1.1, Infrared Data Association, 1995.

[8] IrDA, Serial Infrared Physical Layer Specification for $16 \mathrm{Mb} / \mathrm{s}$ Addition (VFIR) - Errata to version 1.3, Infrared Data Association, 1999.

[9] IrDA: Serial Infrared Link Access Protocol (IrLAP) - Version 1.1, Infrared Data Association, 1996.

[10] IrDA: Serial Infrared Link Access Protocol Specification for $16 \mathrm{Mb} / \mathrm{s}$ Addition (VFIR) - Errata to version 1.1, Infrared Data Association, 1999.

[11] P. Barker, A. C. Boucouvalas, and V. Vitsas, "Performance modeling of the IrDA infrared wireless communications protocol," Int. J. Commun. Syst., vol. 13, pp. 589-604, 2000.

[12] P. Barker and A. C. Boucouvalas, "Performance analysis of the IrDA protocol in wireless communications," in Proc. 1st Int. Symp. Communication Systems Digital Signal Processing, Sheffield, U.K., Apr. 6-8, 1998, pp. 6-9.
[13] W. Bux and K. Kummerle, "Balanced HDLC procedures: A performance analysis," IEEE Trans. Commun., vol. 28, pp. 1889-1898, Nov. 1980.

[14] S. Williams and I. Millar, "The IrDA platform," in Proc. 2nd Int. Workshop Mobile Multimedia Communications, Bristol, U.K., Apr. 11-14, 1995.

[15] V. Vitsas and A. C. Boucouvalas, "Throughput analysis of the IrDA IrLAP optical wireless link access protocol," in Proc. 3rd Conf. Telecommunications, Figueira da Foz, Portugal, Apr. 23-24, 2001, pp. 225-229.

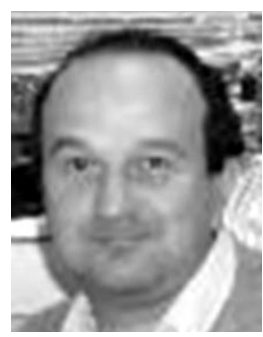

Vasileios Vitsas received the B.Sc. degree in electrical engineering from the University of Thessaloniki, Greece, in 1983 and the M.Sc. degree in computer science from the University of California, Santa Barbara, in 1986. He received the Ph.D. degree in infrared wireless communications from Bournemouth University, Poole, U.K., in 2002.

In 1988, he joined Hellenic Telecommunications Organization where he worked in the field of X.25 packet switching networks. In 1984, he joined the Technological Educational Institution of Thessaloniki, Greece, as a Lecturer in Computer Networks.

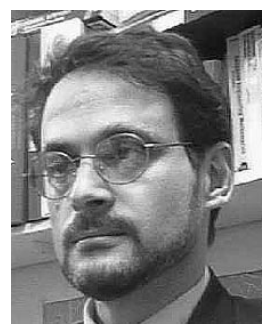

Anthony C. Boucouvalas (S'81-M'82SM'00-F'02) received the B.Sc. degree in electrical and electronic engineering from Newcastle upon Tyne University, U.K., in 1978. He received the M.Sc. and D.I.C. degrees in communications engineering from Imperial College, University of London, U.K., in 1979, and the Ph.D. degree in fiber optics, in 1982.

Subsequently, he joined GEC Hirst Research Centre, and became Group Leader and Divisional Chief Scientist working on fiber-optic components, measurements, and sensors, until 1987, when he joined Hewlett-Packard Laboratories as Project Manager. At Hewlett-Packard, he worked in the areas of optical communication systems, optical networks, and instrumentation, until 1994, when he joined Bournemouth University, Poole, U.K. In 1996, he became a Professor in Multimedia Communications, and in 1999, he became Director of the Microelectronics and Multimedia Research Center. His current research interests lie in optical wireless communications, optical fiber communications multimedia communications, and human-computer interfaces. He has published over 130 papers in the areas of fiber optics, optical fiber components, optical wireless communications, Internet communications, and HCI.

Prof. Boucouvalas is a Fellow of the Inst. Elect. Eng. (FIEE), a Fellow of the Royal Society for the encouragement of Arts, Manufacturers, and Commerce, (FRSA), and a Member of the New York Academy of Sciences and ACM. He is an Editor of the IEEE WIRELESS COMMUNICATIONS MAGAZINE, IEEE TRANSACTIONS ON WIRELESS NETWORKS, EURASIP JOURNAL ON WiRELESS COMMUNICATIONS AND NETWORKS, and Secretary of the IEEE UK\&RI Communications Chapter. He is in the Organizing Committee of the International Symposium on Communication Systems Networks and Digital Signal Processing (CSNDSP), Vice Chair of IEEE GLOBECOM 2003 for Optical Networking and Systems, and a member of the technical committees of numerous conferences. 